\title{
Simulation of Transport Phenomena in Aluminum Nitride Single-Crystal Growth*
}

\author{
Valmor F. de Almeida and J. Carlos Rojo ${ }^{\dagger}$ \\ Nuclear Science and Technology Division \\ OAK Ridge National Laboratory \\ OAK RIDGE, TN 37831-6181, USA
}

Technical Report ORNL/TM-2002/64

2 March 2002

† Vice-President and COO, Crystal IS, Inc., Latham, New York 12110.

*Small CRADA ORNL-00-0587 final report. The Oak Ridge National Laboratory is managed by UT-BATTELLE, LLC for the U.S. Department of Energy under contract DEAC05-00OR22725. This report is also available under request to dealmeidav@ornl.gov or at URL: http://www-smpr.ct.ornl.gov/ ${ }^{\sim}$ dalmeida. 


\begin{abstract}
The goal of this project is to apply advanced computer-aided modeling techniques for simulating coupled radiation transfer present in the bulk growth of aluminum nitride $(\mathrm{AlN})$ single-crystals. Producing and marketing high-quality single-crystals of AlN is currently the focus of Crystal IS, Inc., which is engaged in building a new generation of substrates for electronic and optical-electronic devices. Modeling and simulation of this company's proprietary innovative processing of AlN can substantially improve the understanding of physical phenomena, assist design, and reduce the cost and time of research activities. This collaborative work supported the goals of Crystal IS, Inc. in process scale-up and fundamental analysis with promising computational tools.
\end{abstract}




\section{Contents}

List of figures

List of tables $\quad$ iii

$\begin{array}{lll}1 & \text { Project objectives } & 1\end{array}$

$\begin{array}{lll}2 & \text { Technology background } & \mathbf{1}\end{array}$

$2.1 \quad$ Crystal IS, Inc., interests and efforts in AlN technology . . . . . . . . 4

2.1.1 Crystal growth system design . . . . . . . . . . . 5

2.1 .2 A new crucible technology with lifetimes of over 200 hours . . 5

2.1.3 A method for preparing low-oxygen-content AlN starting material 6

2.1.4 Growth of 13-mm-diameter boules of AlN with large singlecrystal grains .......................... 6

2.1.5 Chemical-mechanical polishing (CMP) . . . . . . . . . . . 9

2.2 Competing AlN substrate manufacturers . . . . . . . . . . . . . . . . 9

2.3 Technology commercialization . . . . . . . . . . . . . . . . . . 12

2.4 Market expectations for GaN-based devices . . . . . . . . . . . . . . 12

2.5 Market price sensitivity . . . . . . . . . . . . . . . . . . . . 14

2.6 Cost of commercial substrates for III-nitride epitaxy . . . . . . . . . . 15

2.7 AlN substrate production cost . . . . . . . . . . . . . . . . 16

3 Radiative-conductive heat transfer in the furnace 17

3.1 Gebhart's net-radiation approach . . . . . . . . . . . . . . . . . . . . 20

3.2 Galerkin-type weak formulation of heat conduction in a multiple regions furnace with discontinuities . . . . . . . . . . . . . . 22

3.3 Finite element approximation . . . . . . . . . . . . . . . . . . . . 24

3.4 Test results . . . . . . . . . . . . . . . . . . . . . . . . . . 27

\begin{tabular}{lll}
\hline 4 & Outlook & 27
\end{tabular}

\begin{tabular}{|ll}
\hline Acknowledgments & 31
\end{tabular}

\begin{tabular}{ll}
\hline References & 31
\end{tabular} 


\section{List of Figures}

2.1 Typical 1-cm-diameter AlN substrate . . . . . . . . . . . . . . . . . 7

2.2 AlN substrate cut from a 13-mm-diameter boule . . . . . . . . . . . 8

2.3 AFM images of the as-grown surface of AlN . . . . . . . . . . . . . . 10

2.4 AFM images of an a-face AlN substrate . . . . . . . . . . . . . . . 11

2.5 Expectation for the GaN market evolution . . . . . . . . . . . . . . . 13

3.1 Components of Crystal IS, Inc. furnace. . . . . . . . . . . . . . . . . 18

3.2 Numbered boundary segments (surfaces) of the furnace. . . . . . . . . 19

3.3 Oriented interface patch with outgoing and incoming radiant fluxes. $~ 19$

3.4 Neighboring domains and their boundary partitions . . . . . . . . . . 22

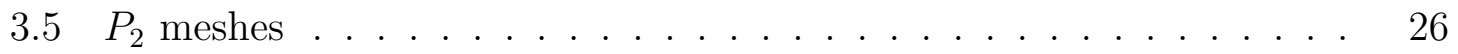

3.6 Temperature field with a 42735 -triangle mesh . . . . . . . . . . . . . 29

3.7 Temperature field with a 101442 -triangle mesh . . . . . . . . . . . . . 30

\section{List of Tables}

2.1 Parameters for possible substrate for nitride epitaxy . . . . . . . . . . 3

3.1 Fictitious physical properties for the furnace . . . . . . . . . . . . . 27

3.2 Boundary conditions of flux and temperature . . . . . . . . . . . . . . 28 


\section{Project objectives}

The immediate objective of this work is to apply continuum models and available 1 simulation capability to aid Crystal IS, Inc., in improving a novel manufacturing process of aluminum nitride. This material, heretofore not produced in commercial scale in the USA nor abroad, is expected to play a significant role in the electronic and optical-electronic industry in the near future (sections 2 and 2.3).

The manufacturing process under development by Crystal IS, Inc., is based on sublimation-recondensation phase change at high temperatures (above $2000^{\circ} \mathrm{C}$ ) within a furnace. Crystal IS, Inc., leads the development of AlN crystal growth technology in the USA and has made significant progress (sec. 2) towards developing a viable process to produce single-crystal bulk aluminum nitride. Crystal IS, Inc., is particularly interested in scaling up the current furnace to produce 50-mm-diameter single-crystals of AlN. Bulk crystals of this size are required as substrates for the next generation of electronic devices. Design and scale-up of a new furnace is the next hurdle to be overcome to show commercial viability of the manufacturing process. This represents a prime opportunity to apply modeling and computational methods to accelerate and reduce the cost of the development of a novel and important industrial process.

The proposed modeling (sec. 3) takes into account non-linear continuum radiative heat transfer at high temperatures to compute accurate temperature gradients in the growth zone within the furnace, which is critical for crystal quality and process control. Since thermal radiation is the dominant mode of heat transfer in the furnace, a diffusive gray-body thermal radiation model is initially employed. This task entails solving the heat conduction equation with meticulous accounting of the axisymmetric geometry of the furnace so that configurational factors in various structural elements are correctly computed. This approach was reviewed and discussed with Crystal IS, Inc., and considered promising in view of the features of the furnace. Future model validation, through experimental results of crystal composition and morphology provided by Crystal IS, Inc., will determine the viability of process design through computer simulation.

\section{Technology background}

Semiconductors based on gallium nitride $(\mathrm{GaN})$ are expected to play an increasingly important role in the electronic and optical-electronic industry (sec. 2.3 and fig. 2.5). The current worldwide development of III-nitride epitaxy has opened new possibilities for this semiconductor in numerous applications, including blue/UV solid stated charge injection lasers, UV optical sources and detectors, high-power microwave devices, high-power switches, high-temperature devices, high-density optical data stor-

Oak Ridge National Laboratory Technical Report ORNL/TM-2002/64 — pp. 1-32 
Valmor de Almeida and J. Carlos Rojo

age, and wireless communication. However, there still exists no cost-effective substrate for constructing these devices. Even for cost-insensitive buyers, the use of these revolutionary devices will be severely restricted until the devices become widely used in commercial service. That requires an economically efficient (sec. 2.3p) substrate such as the one Crystal IS, Inc., aims to develop.

In the aforementioned applications, layers of nitride semiconductors are deposited onto a single-crystal substrate. In addition to mechanical strength, a satisfactory substrate needs to match the crystal structure and lattice parameter closely, and be chemically compatible with the device layers grown on it. Ideally, the thermal conductivity of the substrate should be high to dissipate heat generated by the active device, and the thermal expansion should match that of the semiconductor.

One of the most important problems that has kept the blue/UV laser diodes (LD), based on III-nitride technology, from the high volume market is the absence of an ideal substrate (the market for nitride-based LDs is estimated to be 10 billion US\$/year in this decade; sec. 2.3). Sapphire and $\mathrm{SiC}$ are the only two substrates that are currently being used for the production of commercial GaN-based devices such as blue and violet light-emitting diodes (LEDs) (using $\mathrm{SiC}$ and sapphire) or blue-violet LDs (using sapphire).

Many manufacturers have chosen sapphire because high-quality, inexpensive substrates are commercially available. However, sapphire is far from being an ideal substrate for GaN epitaxy. Its lattice mismatch to $\mathrm{GaN}$ is enormous (about 16\%), it has little distinction between the + and - [0001] directions which can give rise to +/c-axis domains in epitaxial films of $\mathrm{GaN}$, and its differential thermal expansion can lead to cracking during the cooling process after the device fabrication process. In spite of those problems, recently, Nichia Ltd. (Japan) has announced the production of the first violet laser with commercial possibilities (more than 10,000 hours of operating life) using sapphire substrates. The LDs were initially offered for around $\$ 2,000$ a unit. Using sapphire substrates leads to a costly fabrication process since it requires growing buffer layers and using Lateral Overgrowth Techniques (LOT). Because sapphire has a low thermal conductivity that traps heat from the LD, Nichia and others are using freestanding substrates for higher power LD structures. In this technique, the substrate is removed after a thick GaN layer is grown atop the sapphire. This method leaves the GaN as the base for building the laser. This base is much better at dissipating heat (table 2.1) and it matches the lattice of the alloy layers perfectly. However, this alternative will certainly increase fabrication costs.

Single-crystal substrates of $\mathrm{SiC}$ are attractive due to their close lattice match to $\mathrm{AlN} / \mathrm{GaN}$ in the plane perpendicular the c-axis (the so-called c-plane) and very high thermal conductivity (its theoretical value is $4.9 \mathrm{~W} / \mathrm{cm}-\mathrm{K}$ although typical substrates are $\approx 3 \mathrm{~W} / \mathrm{cm}-\mathrm{K}$ ). In addition, $\mathrm{SiC}$ substrates can be made electrically conducting, which is very attractive for some applications (such as LEDs and LDs). However, $2 \mathrm{H}$

Oak Ridge National Laboratory Technical Report ORNL/TM-2002/64 — pp. 1-32 
Table 2.1: $\quad$ Parameters for possible substrate alternatives for nitride epitaxy.

\begin{tabular}{|c|c|c|c|c|c|}
\hline & GaN & AlN & 4H-SiC & $6 \mathrm{H}-\mathrm{SiC}$ & Sapphire \\
\hline \hline $\begin{array}{c}\text { Crystal } \\
\text { Structure }\end{array}$ & $\begin{array}{c}\text { hexagonal } \\
(2 \mathrm{H})\end{array}$ & $\begin{array}{c}\text { hexagonal } \\
(2 \mathrm{H})\end{array}$ & $\begin{array}{c}\text { hexagonal } \\
(4 \mathrm{H})\end{array}$ & $\begin{array}{c}\text { hexagonal } \\
(2 \mathrm{H})\end{array}$ & rhombohedral \\
\hline Bandgap $(\mathrm{eV})$ & 3.39 & 6.2 & 3.26 & 3.03 & 9.9 \\
\hline $\begin{array}{c}\text { Lattice } \\
\text { Constant }(\AA)\end{array}$ & $\begin{array}{c}a=3.189 \\
c=5.185\end{array}$ & $\begin{array}{c}a=3.11 \\
c=4.987\end{array}$ & $\begin{array}{c}a=3.073 \\
c=10.053\end{array}$ & $\begin{array}{c}a=3.081 \\
c=15.117\end{array}$ & $\begin{array}{c}a=4.76 \\
c=12.99\end{array}$ \\
\hline $\begin{array}{c}\text { Thermal cond. } \\
(\mathrm{W} / \mathrm{cm}-\mathrm{K})\end{array}$ & 1.7 & 3.2 & 4.9 & 4.9 & 0.35 \\
\hline
\end{tabular}

$\mathrm{SiC}$ (which is the crystal structure that $\mathrm{GaN}$ and $\mathrm{AlN}$ have) is not available and the lattice mismatch along the c-axis for both $4 \mathrm{H}$ and $6 \mathrm{H} \mathrm{SiC}$ is substantial. In addition, the chemical bonding between the Group-IV elements of the $\mathrm{SiC}$ and the Group-III or Group-V elements of the nitrides is expected to create potential nucleation problem and will certainly lead to electronic states at the interface.

For devices that use $\mathrm{GaN}$ or $\mathrm{Ga}_{1-x} \mathrm{In}_{x} \mathrm{~N}$, the best possible substrate would be large-area GaN single-crystal wafers. Several methods to grow bulk GaN crystals have been proposed. While this possibility has become more attractive in the last few years, it does not appear to be commercially feasible to fabricate large bulk crystals of $\mathrm{GaN}$ in the near term.

The importance of nitride technology has triggered a search for other substrates such as $\mathrm{AlN}, \mathrm{ZnO}, \mathrm{LiGaO}_{2}, \mathrm{Mg} \mathrm{Al}_{2} \mathrm{O}_{4}$, or $\mathrm{Si}$, although, at this point in time, there has not been any nitride commercial device fabricated on any such alternative substrate. Aluminum nitride is very attractive because it has a relatively small lattice mismatch (around 2.2\%) with GaN, and it has almost constant thermal expansion at temperatures ranging from the ambient to $1000{ }^{\circ} \mathrm{C}$. It also has the same wurtzite $(2 \mathrm{H})$ crystal structure as GaN and the same kind of piezoelectric polarity. Also, the chemical compatibility with GaN is much better than that of the SiC. Last but not least, AlN substrates will be more attractive for $\mathrm{Al}_{x} \mathrm{Ga}_{1-x} \mathrm{~N}$ devices requiring higher $\mathrm{Al}$ concentration that are attractive for high-temperature, high-power, radiation hardened, and UV wavelength applications. 
Valmor de Almeida and J. Carlos Rojo

\subsection{Crystal IS, Inc., interests and efforts in AlN technology}

Crystal IS, Inc., was founded in 1997 and is focused on the production of commercial AlN single-crystal substrates cut from bulk crystal boules. Their effort was inspired by an earlier investigation carried out by G. A. Slack (one of the co-founders) and T. McNelly in the late 70's. The investigation demonstrated the crystal growth of AlN using the sublimation-recondensation technique at $2250{ }^{\circ} \mathrm{C}$ in a gas mixture of $95 \% \mathrm{~N}_{2}$ and $5 \% \mathrm{H}_{2}$ at a maximum growth rate of $0.3 \mathrm{~mm}$ per hour. At the time this technique was developed, the size of the AlN boules was limited by the lifetime of the crucibles. This key issue prevented further work on increasing the AlN boule size. Crystal IS was founded with several innovative ideas that promised to overcome this barrier. As indicated below, the feasibility of constructing cost-effective crucibles has been demonstrated as a result of a Phase I STTR (with Rensselaer Polytechnic Institute) funded by the Ballistic Missile Defense Organization, DoD (BMDO). This STTR was entitled Innovative Crucible Design for Commercial Growth of Aluminum Nitride, (contract \# N00014-97-C-0362) and was monitored by the Office of Naval Research (ONR), Dr. Colin Wood. Crystal IS, Inc., has been awarded two additional SBIRs that have boosted the AlN substrate development effort. The first was a Phase I SBIR from ONR entitled Development of Commercially Viable Growth Rates for Bulk Aluminum Nitride Crystals, (contract \# N00014-98-C-0053, monitored by Dr. Colin Wood) and the second was a Phase I SBIR funded by BMDO and entitled Preparation of Aluminum Nitride Substrates for Device Fabrication. The latter Phase I SBIR was administered by the Air Force Research Laboratory (AFRL contract \# F33615-98-C-1325, administered by Thomas Wille with technical point of contact, Dr. Cole Litton). The Phase II proposal has been submitted as a result of the successful completion of the BMDO Phase I SBIR (contract \# F33615-98-C-1325) and it combined all three prior STTR/SBIR efforts. Further research has been funded by private investment by the company co-founders Leo Schowalter, Glen Slack, and J. Carlos Rojo.

The research and development activities of Crystal IS are two-fold: crystal growth and preparation of substrates. A number of $R \& D$ achievements have positioned Crystal IS as a worldwide leader in AlN substrate development. Notably,

1. Furnace technology needed for AlN crystal growth,

2. A method for preparing low oxygen content AlN starting material,

3. A new crucible technology with lifetimes of over 200 hours, (BMDO STTR, monitored by ONR),

4. A chemical mechanical polishing (CMP) technique capable of preparing atomically smooth surfaces of AlN suitable for epitaxial growth. (BMDO SBIR,

Oak Ridge National Laboratory Technical Report ORNL/TM-2002/64 — pp. 1-32 
monitored by AFRL),

5. Growth of $13 \mathrm{~mm}$ diameter boules of AlN with large single-crystal grains. (Navy SBIR in collaboration with Rensselaer Polytechnic Institute).

Each of these developments is described in greater detail below. We should emphasize that the crystal growth technology of Crystal IS, Inc., is under experimental stage and further work is necessary to bring it to commercial production. The technical risk associated with achieving a reliable, reproducible, and cost-efficient process to grow 50-mm-diameter boules, which meets the specifications of the device manufacturing industry, is still substantial. The design of a new furnace of larger capacity is the next hurdle to be overcome.

The highly non-linear transport phenomena taking place in the existing furnace prevent a straightforward scale-up calculation. Therefore the ultimate purpose of this project is to employ computer-aided modeling to guide the design of the new furnace.

\subsubsection{Crystal growth system design}

During the growth of AlN by the sublimation-recondensation technique, many elements of the system run at temperatures over $1900{ }^{\circ} \mathrm{C}$. Therefore, the choice of materials and components becomes a delicate matter. Crystal IS, Inc., has designed a radio-frequency furnace and has showed that $\mathrm{AlN}$ boules can be grown up to $13 \mathrm{~mm}$ in diameter. Important fundamental understanding of growth conditions, transport phenomena and growth kinetics has been gained with this system. Lately, a furnace of similar capacity has been designed and operated with resistance heating in different zones. This allows the control of the temperature of different components in the furnace and provides experimental data for validation of theoretical models.

\subsubsection{A new crucible technology with lifetimes of over 200 hours}

During the growth process, the crucible has to survive the corrosive interaction with $\mathrm{Al}$ in vapor phase at $2000{ }^{\circ} \mathrm{C}$ for the entire duration of the process. Ideally the crucible should have a lifetime spanning several runs. In the early work of SLACK AND MCNELLY (1976) (SLACK AND MCNELLY, 1977), CVD (chemical vapor deposition) polycrystalline tungsten was found to be adequate to perform short runs and grow 0.5 -cm-diameter single-crystals. However, long runs were not possible. After several hours, the $\mathrm{Al}$ vapor penetrated in the crucible walls, resulting in disintegration and catastrophic failure of the process. Crystal IS, Inc., led a BMDO STTR Phase I effort to design and test several possible crucibles to allow large diameter AlN crystal boules to be grown. The result of this investigation was a proprietary crucible technology that can stand the $\mathrm{Al}$ vapor attack.

Oak Ridge National Laboratory Technical Report ORNL/TM-2002/64 — pp. 1-32 
Valmor de Almeida and J. Carlos Rojo

\subsubsection{A method for preparing low-oxygen-content AlN starting material}

In order to grow high-purity AlN crystals, it is necessary to have a source of highpurity starting material. Crystal IS, Inc., uses polycrystalline AlN. However, the best AlN sold commercially is a powder that contains a minimum of $0.5 \%$ of oxygen by weight. This material is inadequate for crystal growth. The oxygen will provoke the earlier evaporation of oxynitrides with higher vapor pressures than AlN at low temperatures. This is detrimental to the nucleation process. Other significant adverse effects of the oxygen is the reduced thermal conductivity and contamination, suspected to play a role in the formation of stacking faults in the AlN crystal. Furthermore, the formation of electronic levels in the middle of the AlN band gap will preclude the development of a desirable doping process. Crystal IS, Inc., has successfully developed a proprietary method to obtain high purity polycrystalline AlN that satisfies the demand of material for the growth runs to be performed during this investigation.

\subsubsection{Growth of 13-mm-diameter boules of AlN with large single-crystal grains}

Several factors can provoke deleterious effects on the nucleation process:

1. Quality of the starting material (the earlier formation and evaporation of oxynitrides can contribute to a malnucleation),

2. Inadequate cleaning process of the crucible internal walls (failure to remove residues can contribute to an inhomogeneous nucleation), and

3. Thermal maldistribution causing a large supersaturation leading to an uncontrollable nucleation process along the crucible walls.

These factors were controlled in order to enhance the nucleation process.

Figures 2.1 and 2.2 show two typical substrates currently produced at Crystal IS, Inc. Although Crystal IS, Inc. has produced single-crystal boules with diameter exceeding $15 \mathrm{~mm}$, thermal cracking precludes the possibility of obtaining a full diameter substrate wafer after cutting and polishing. This is a critical issue that needs to be addressed to scale up the size of existing substrates. Numerical modeling of the thermal environment inside the system will be of paramount importance to understand and prevent the cracking of the single-crystal boules.

AFM (atomic force microscope) imaging (fig. 2.3) of an AlN as-grown surface reveals interesting information about the growth mechanism of AlN. It is observed that, consistent with the x-ray analysis, the growth front atomic arrangement (fig. 2.3p) corresponds to the characteristic structure of c-face fronts of hexagonal wurzite-type

Oak Ridge National Laboratory Technical Report ORNL/TM-2002/64 — pp. $1 \sqrt{32}$ 


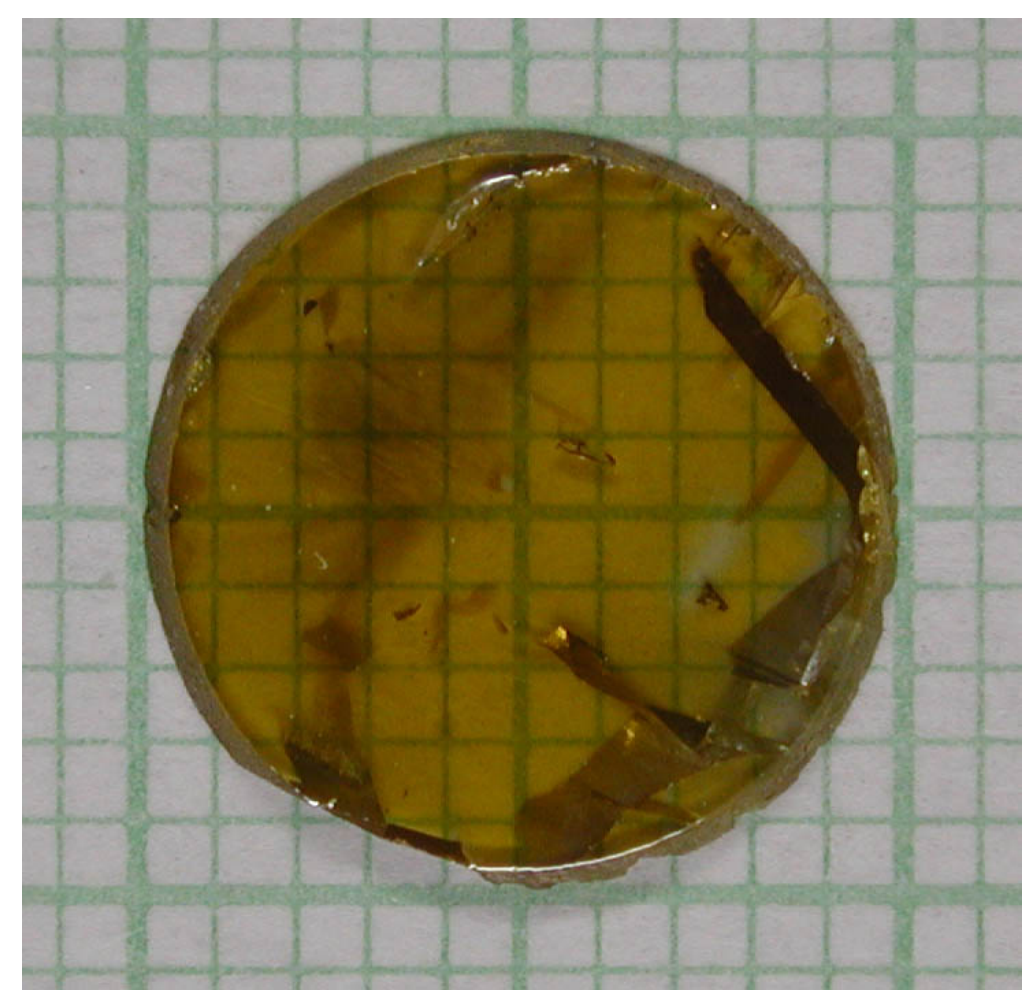

Fig. 2.1: Typical 1-cm-diameter AlN substrate. A severe crack can be observed on the right side of the substrate. The left size was crack-free, but X-ray topography revealed a highly stressed substrate. 


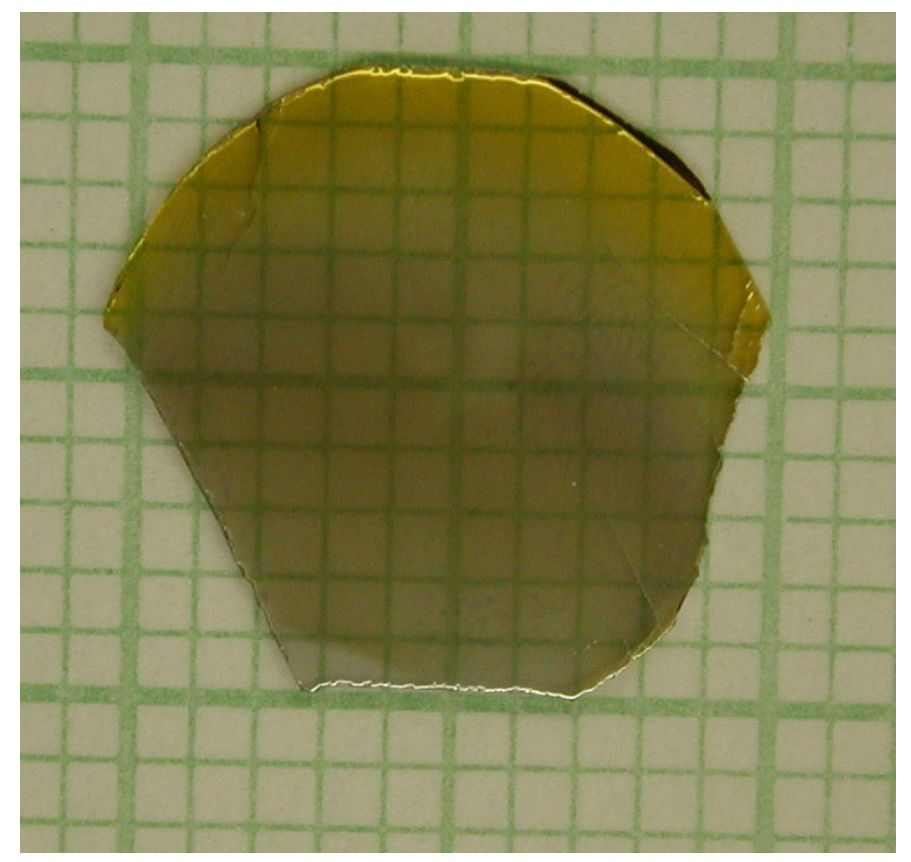

Fig. 2.2: Substrate cut from a 13-mm-diameter boule. As a result of a crack, the substrate did not exceed $1 \mathrm{~cm}$ in diameter. However, this substrate was stress-free with an excellent crystalline quality. 
crystals. The straight segments of the steps indicate that the migration length of atoms along the steps is greater than a micrometer. The diffusion length of the atoms on the terraces must be even higher than that. The origin of the step flow is related to several outlets of screw dislocations (fig. 2.3a). The density of the screw dislocations has been estimated to be $\approx 5104 \mathrm{~cm}^{2}$. Their step edges have the shape of the Archimedes spiral without distinct straight segments; the crystal growth is governed not only by the diffusion but also by the strain field of the dislocation.

\subsubsection{Chemical-mechanical polishing (CMP)}

The use of AlN as substrate for device fabrications requires necessarily the development of a technology to adequately prepare the AlN surface for epitaxial growth. Due to the AlN substrate unavailability at the time Crystal IS, Inc., started operating, a $\mathrm{R} \& \mathrm{D}$ program was conducted to eliminate the uncertainty about how to prepare $\mathrm{AlN}$ substrates for epitaxy. Epitaxial nitride layers were grown on the substrates by collaborators at the Rensselaer Polytechnic Institute. One of the main achievements of Crystal IS, Inc., has been the development of proprietary chemical-mechanical polishing (CMP) techniques. Examination of substrate by AFM (fig. 2.4) after CMP shows a nearly atomically flat surface without any evidence of mechanical damage (sawing damage, scratches, etc.) Epitaxial growth of $0.7-\mu \mathrm{m}$-thick AlN and $1-\mu \mathrm{m}$-thick $\mathrm{Al}_{0.5} \mathrm{Ga}_{0.5} \mathrm{~N}$ epitaxial layers, and Rutherford backscattering/ion channeling spectra were used to determine the crystal quality of the epitaxial layers.

After growth of a $0.7-\mu \mathrm{m}$-thick homoepitaxial layer of $\mathrm{AlN}$, the channeling minimum yield $\chi_{\text {min }}$ remained $1.5 \%$. The $\chi_{\text {min }}$ measured for the $\mathrm{Al}_{0.5} \mathrm{Ga}_{0.5} \mathrm{~N}$ layer was $2.2 \%$, which is still excellent considering that no attempt was made to optimize the growth parameters. This excellent ion channeling indicates that the surface obtained by the CMP process is adequate to perform III-nitride epitaxy on those substrates. Crystal IS, Inc., has demonstrated (KING et al., 1996; LU et al., 1998; STEIGERWALD et al., 1997) that high quality a-face AlN substrates can be prepared from a bulk single-crystal of $\mathrm{AlN}$. In addition, high quality epitaxial layers of $\mathrm{AlN}$ and $\mathrm{Ga}_{1-x} \mathrm{Al}_{x} \mathrm{~N}$ have been created without the need for buffer layers. These substrates should prove very attractive for nitride device fabrication in the future due to their high thermal conductivity and minimal thermal expansion mismatch between $\mathrm{AlN}$ and GaN.

\subsection{Competing AlN substrate manufacturers}

The need for adequate substrates or alternative technologies for III-nitride epitaxy and the dimension of the potential market (sec. 2.3) for this product have attracted several companies and universities involved in the development of substrates. Crystal

Oak Ridge National Laboratory Technical Report ORNL/TM-2002/64 — pp. 1-32 

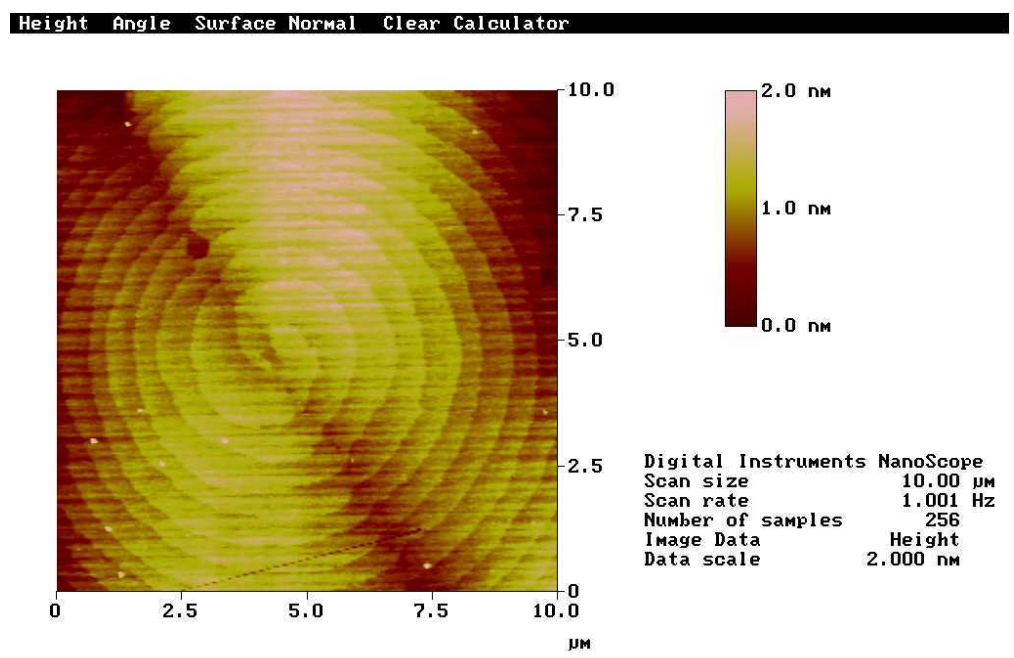

Digital Instruments NanoScope Scan size $10.00 \mathrm{jM}$ Scan rate $\quad 1.001 \mathrm{~Hz}$ Number of samples Height $\begin{array}{lr}\text { Image Data } & \text { Height } \\ \text { Data scale } & 2.000 \mathrm{~nm}\end{array}$

Sample 03 alN free grown c-facet.

aln $03-38.001$ Height

(a)
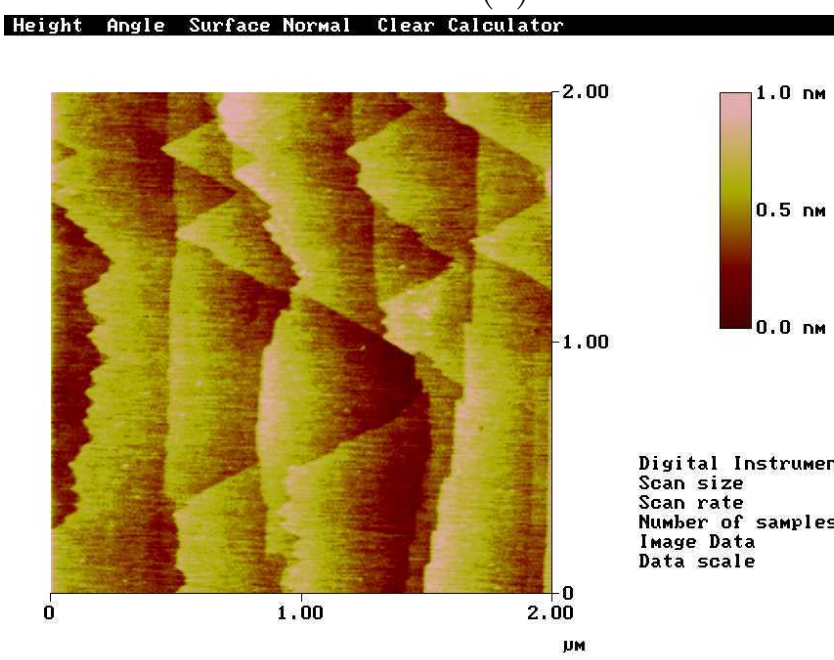

Digital Instruments NanoScope Scan size 2.000 $\begin{array}{ll} & 2.001 \\ \text { Scan rate }\end{array}$ Number of samples 256 Image Data Height Sample 03 AlN free grown c-facet
aln03-21.001 Height

(b)

Fig. 2.3: AFM images of the as-grown surface of AlN. (a) Screw dislocation. (b) Step flow far away from the dislocation.

Oak Ridge National Laboratory Technical Report ORNL/TM-2002/64 — pp. 1-32 


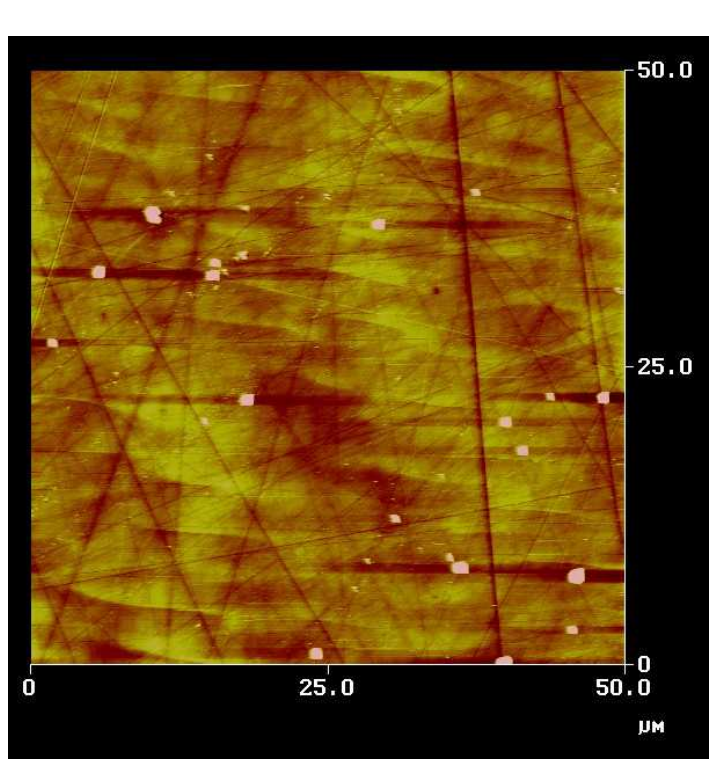

(a)

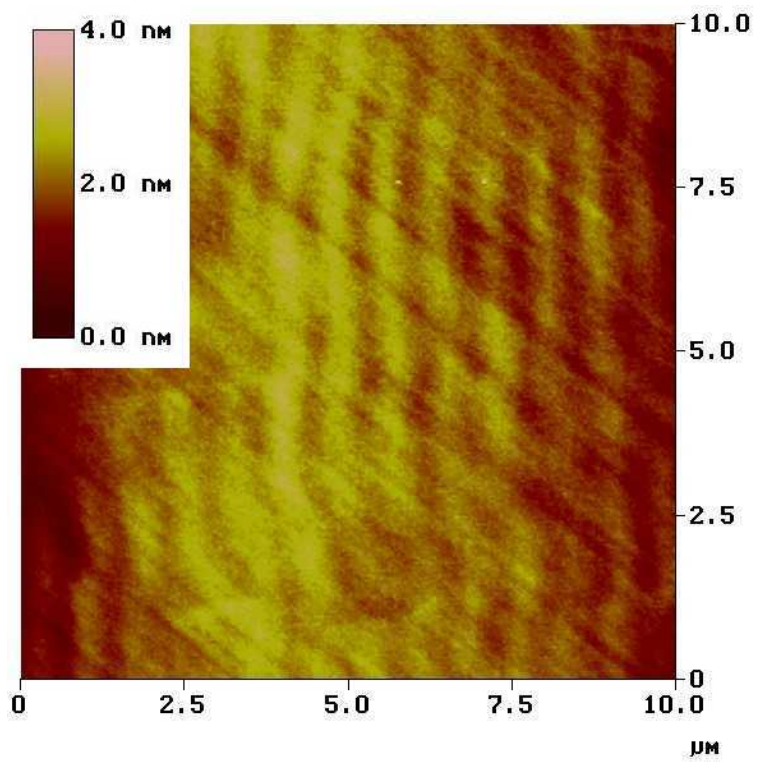

(b)

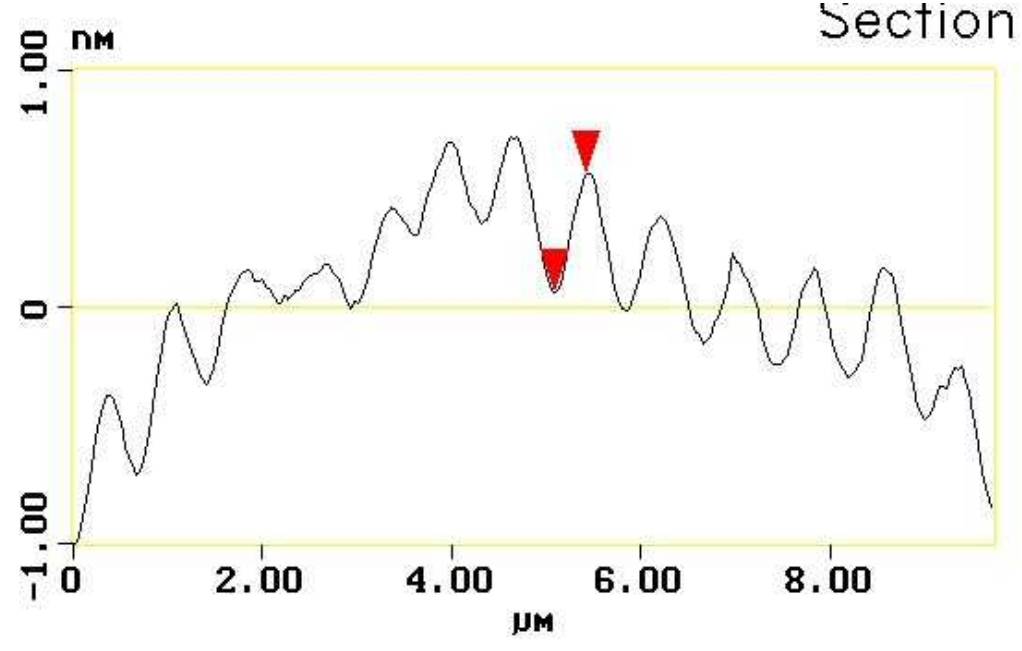

(c)

Fig. 2.4: AFM images of an a-face AlN substrate. (a) Diamond polishing only. (b) Proprietary CMP process. (c) Profile across the AFM image showing the atomic height steps of $0.25 \mathrm{~nm}$. 
IS, Inc., is currently competing with several other endeavors: Cornell University, Cermet Inc., North Carolina State University, and Kyma Inc.

Crystal IS, Inc., is leading the AlN single-crystal growth technology as was evident in a recent review of bulk nitride crystal growth workshop held at the Naval Research Laboratories (Nov. 22 and 23, 1999). At that time, Crystal IS, Inc., showed its 13mm-diameter, 15-mm-long boule growth capability and the results of epitaxial growth of $\mathrm{AlN}$ and $\mathrm{Al}_{x} \mathrm{Ga}_{1-x} \mathrm{~N}$ on a-face substrates. Crystal IS, Inc., has demonstrated superior material quality to all other AlN competitors.

\subsection{Technology commercialization}

The current worldwide development of III-nitride epitaxy has opened many new innovative possibilities for wide bandgap and high temperature semiconductors. However, there still exists no economically efficient substrate. Until such a substrate has been developed, the world of blue/UV solid-state charge injection lasers, UV optical sources and detectors, high-power microwave devices, high-power switches, and hightemperature applications will not get beyond the cost-insensitive buyers. But, even for those buyers, the use of these revolutionary devices will be severely restricted until the devices become widely used in commercial service. That requires an economically efficient substrate such as the one in development by Crystal IS, Inc. The combined experimental-modeling-computational effort of this project is crucial in making $\mathrm{AlN}$ single-crystal substrates economically feasible.

Below, we provide a more detailed synopsis of the current state of nitride technology, the role substrates play in the current development, and the potential for this technology if an appropriate substrate technology is developed.

\subsection{Market expectations for GaN-based devices}

Emerging technology based on GaN is expected to play an increasingly important role in the optical-electronic industry and to grow significantly during the next decade (fig. 2.5). Among GaN device applications, blue-UV LED (light-emitting diodes) and LD (laser diodes) will represent a large fraction of the total LD and LED market, which is estimated to be $\$ 4.5$ billion in 2002 (Compound Semiconductor 1999 vol. 5, March ).

Nichia (Japan) has estimated that the market for nitride-based LDs alone will become $\$ 10$ billion per year during the coming decade assuming that the market price can be reduced from $\$ 2,000 /$ unit (price in 1999) to $\$ 10 /$ unit. This prediction is actually fairly conservative given the growing demand for inexpensive, high-density data storage generated by burgeoning information technology field. By comparison, in 1999, the consumption of LDs was estimated to be 300 million units. While scaling

Oak Ridge National Laboratory Technical Report ORNL/TM-2002/64 — pp. 1-32 


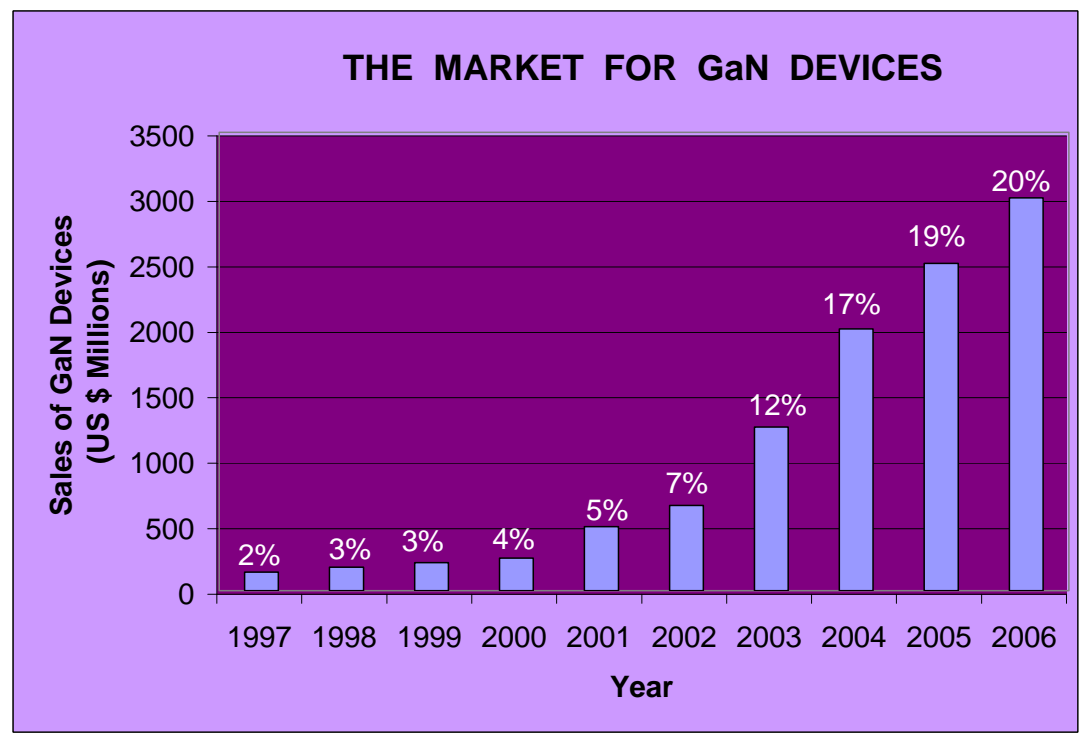

Fig. 2.5: Expectation for the GaN market evolution. Percentages shown are predicted fraction of the total compound semiconductor market. Source: A. R. Miles 1998 IIIVs Review 11(1) p. \%. Report from Strategies Unlimited. 
Valmor de Almeida and J. Carlos Rojo

current fabrication technology to 1 billion LDs/yr should decrease the cost of these LDs, the $\$ 10 /$ unit target is unrealistic without the introduction of a new substrate technology.

On the order of 10,000 LDs can be fabricated on a 50-mm-diameter substrate if sufficiently high yield is achieved. Thus, the processing costs to fabricate LDs must be substantially less than $\$ 100,000$ /substrate to make a profit at the $\$ 10 /$ unit price level. This is the key question to be answered for any new substrate technology proposed for this market. What yield is achievable and what will the resultant total processing cost be? Based on past experience with new semiconductor technology, substrate costs of 1 to $5 \%$ of the final product cost are acceptable. Therefore, a $\$ 10$ billion LD market will generate a substrate market ranging from $\$ 100$ million to $\$ 450$ million for this application alone. This would correspond to a market need of at least 100,000 $50 \mathrm{~mm}$ diameter substrates per year for just the blue/UV LD market. Substrate prices of less than $\$ 4,000$ should prove attractive if, in fact, it allowed a $\$ 10 /$ LD manufacturing process to be developed. Demonstration of this capability in AlN substrate development is the key risk to Crystal IS, Inc. As discussed below, we have estimated the cost of producing $50 \mathrm{~mm}$ diameter AlN substrates to be less than $\$ 700$ for a mature technology.

Of course, such a large and mature market for nitride LDs would also allow other businesses to take advantage of nitride high-power and high-temperature capabilities at markedly reduced prices per unit of performance. Thus, price-insensitive buyers would also benefit from nitride technology for propulsion applications, for space electronics where radiation hardening is imperative, and for UV solid-state emitters and detectors needed for missile defense, optical communications and data storage.

\subsection{Market price sensitivity}

The cost of fabrication is a main issue of concern for the device manufacturers for any electronic or optical-electronic application related to the large-volume production, such as LDs for storage applications. When a new technology is in a development stage or it is focused on supplying high performance devices to price-insensitive customers, the fabrication cost is expected to be very high. In this case, even if the substrate is very expensive, its cost will not represent a large percentage of the total device fabrication cost. However, in order to reach the high-volume market, the device fabrication cost has to be substantially decreased and, as a general rule, the cost of the substrate has to be no more than 1 to $5 \%$ of the total cost of the device. This means that the substrate cost is limited to be less than $5 \%$ of the number of operating devices obtained per substrate (yield) multiplied by the total fabrication cost of one device unit. This objective can only be achieved if the substrate meets two requirements. First, it is necessary to have a mature crystal growth technology that

Oak Ridge National Laboratory Technical Report ORNL/TM-2002/64 — pp. $1 \sqrt{32}$ 
allows a reproducible fabrication process of large area (at least $50 \mathrm{~mm}$ ) substrates. Second, the quality of the substrates has to lead to a high yield that optimizes the fabrication cost per device for a given fixed cost of running a substrate through the device fabrication process. However, the availability of large, cheap substrates, which allows a relatively high yield, does not always guaranty the possibility of fabricating inexpensive devices. If the substrate characteristics require a very complex and costly process to achieve a large yield, the substrate cost becomes insignificant compared to the high fabrication costs, which will prevent these devices from creating a highvolume market for price-sensitive customers.

\subsection{Cost of commercial substrates for III-nitride epitaxy}

The III-nitride technology is still in its earliest stages. However, due to the extraordinary variety of potential applications, there is enormous interest to develop this technology. Thus, during the last five years many SBIR and STTR programs have been granted to companies involved in the development of III-nitride electronic and optical-electronic devices. One of the main problems that this development effort has found is the lack of an adequate substrate for III-nitride epitaxy. This issue explains the number of SBIR and STTR DoD programs dedicated to substrate fabrication for III-nitrides (i.e., American Xtal, Cermet, Crystal IS, Inc.).

Currently, only two substrates are being used to develop GaN commercial devices: SiC (blue LEDs) and sapphire (blue LEDs, UV detectors, and LDs). The main motivation for using sapphire is the high stability and the availability of cheap material (a typical 50-mm-diameter substrate costs $\$ 120$ ). However, this substrate is not at all an ideal substrate for III-nitride so that the manufacturing of devices based on sapphire requires complex processing that increases the cost of production. While Nichia (Japan) is already marketing a violet III-nitride laser for $\$ 2,000$, they publicly recognize that only by reducing this cost to around $\$ 10$ per LD will it be possible to reach the potential $\$ 10$ billion/year blue/UV LD market estimated for this decade. Given the current commercial price, the substrate cost is completely negligible. This could be mainly due to the immature fabrication process and the complexity added by the use of a clearly inadequate substrate. While $\mathrm{SiC}$ wafers are approximately five times more expensive (last fall, Cree did announce the commercialization of a 50-mm-diameter $\mathrm{SiC}$ wafer for $\$ 495$ ), this does not represent a problem for the use of $\mathrm{SiC}$ in developing a commercial blue-UV LD if a high yield, less expensive process can be developed. However, no long lifetime blue-violet laser has been produced on $\mathrm{SiC}$ yet.

Both the high cost of fabrication using sapphire and the absence of a blue/UV laser based on $\mathrm{SiC}$, strongly suggests the need to bring alternative, more adequate substrate from the research laboratories to a production stage. Even though GaN

Oak Ridge National Laboratory Technical Report ORNL/TM-2002/64 — pp. 1-32 
Valmor de Almeida and J. Carlos Rojo

would be the ideal substrate for fabrication of GaN-based LDs, and AlN exhibits physical-chemical properties much more appropriate than those of sapphire or $\mathrm{SiC}$, manufacturers take into consideration the availability of a specific material to be used in a fabrication process. Before private capital investors use millions of dollars in the $R \& D$ of development of devices based on a substrate, they want to be sure that positive results of their development will lead to a production process. This is only possible if (a) there is a mature growth technology for the specific substrate they are using, and (b) the cost of the substrate, assuming a mature growth technology, is $1-5 \%$ of the fabrication cost necessary to generate multi-million sales. Both GaN and AlN will require an initial kickoff investment to demonstrate that it is possible to transform laboratory experiments into efficient production processes which will eliminate most of the risk and attract the private capital. This capital will bring the process to a mass production process. At this point, only programs such as SBIR, STTR, LTR, etc., can justify the investment on this high-risk initial effort.

\subsection{AlN substrate production cost}

At this time, Crystal IS, Inc., has demonstrated the possibility of growing 13-mmdiameter AlN boules. However, the quality of these boules can be substantially improved. In order to attract private investors and give another guaranteed alternative substrate to device manufacturers, we will need to demonstrate substrates that are at least $25-50 \mathrm{~mm}$ in diameter with a better quality level than those achieved so far.

The large investment required for building a larger furnace capable of growing 50mm-diameter AlN single crystals compels the use of computational modeling, guided by laboratory experiments on existing furnaces, to reduce costs and time-consuming trial-and-error approaches for scale-up.

Assuming the growth technology will not be dramatically modified with respect to what Crystal IS, Inc., has developed so far, the cost of all the steps and tasks necessary to produce a 50-mm-diameter substrate can be divided into four major sections comprising: starting material, crucible, labor, and fixed. The last section includes the equipment, rent, energy, substrate preparation, etc. We estimate that assuming a mature growth technology for 50-mm-diameter AlN boules, 50-mm-diameter substrates can be produced at a competitive cost compared to that of the $\mathrm{SiC}$ substrates.

Oak Ridge National Laboratory Technical Report ORNL/TM-2002/64 — pp. 1-32 


\section{Radiative-conductive heat transfer in the fur- nace}

Crystal IS, Inc., AlN single-crystal growth technology is proprietary. Some components of a given axisymmetric furnace are shown in figure 3.1 and labelled as computational regions from 1 to 7 . It is desirable to be able to predict the steady-state temperature field in the regions. As a first approximation, attention is given to the problem of couple radiative-conductive heat transfer. The empty space, region 1 , (not necessarily a region under vacuum) is considered as a nonparticipating medium, that is perfectly transparent (non-absorbing, non-emitting, and non-scattering). The external walls and heating elements are taken to be at a constant temperature. All regions, with exception of the empty space, are supposed to be opaque, gray, and diffusive, that is, the radiant energy emitted is independent of the frequency of the radiation and independent of the direction of the beam. The inhomogeneity of the temperature field within the regions is due to molecular thermal conduction. Therefore the system could be examined by first calculating the radiative-conductive heat transfer in the regions 2 to 7, independently from the empty space; and second, by "post-processing" the temperature field in the empty space by solving a heat conduction equation in the empty space domain. The solution method chosen here ignores this two-step analysis approach and treats the problem in a fully coupled fashion.

The domain of interest accepts different ways of partitioning its boundary. In figure 3.2 a preferred partition was made and represented by numbering various segments to allow for convenient application of required boundary conditions. The boundary segments $4,9,10,11$, and 12 , constitute the axis of symmetry of the domain and the boundary condition there is of null heat flux. The dividing segments between regions are called interfaces and are automatically labelled as a function of the numbering of adjacent regions. On region interfaces, there is always a non-zero heat flux with contributions from conduction and radiation transfer as described next.

Let $\Gamma_{N, e}$ be an oriented surface patch element of an interface with area $A_{e}$ and unit normal field $\boldsymbol{n}_{e}$ (fig. 3.3). The pointwise outgoing and incoming radiant fluxes per unit of time are designated by $\boldsymbol{q}_{i, e}$ and $\boldsymbol{q}_{o, e}$ with the following sign convention for the respective magnitude fields

$$
q_{o, e}(\boldsymbol{x}):=\boldsymbol{q}_{o, e}(\boldsymbol{x}) \cdot \boldsymbol{n}_{e}(\boldsymbol{x}) \geq 0 \quad \text { and } \quad q_{i, e}(\boldsymbol{x}):=-\boldsymbol{q}_{i, e}(\boldsymbol{x}) \cdot \boldsymbol{n}_{e}(\boldsymbol{x}) \geq 0
$$

where $\boldsymbol{x}$ is a point on $\Gamma_{N, e}$. Therefore the net flux leaving the patch element $\boldsymbol{q}_{e}$ is

$$
\boldsymbol{q}_{e}(\boldsymbol{x}):=\boldsymbol{q}_{o, e}(\boldsymbol{x})+\boldsymbol{q}_{i, e}(\boldsymbol{x}) \quad \text { or in magnitude form } \quad q_{e}(\boldsymbol{x}):=q_{o, e}(\boldsymbol{x})-q_{i, e}(\boldsymbol{x}) \text {. }
$$




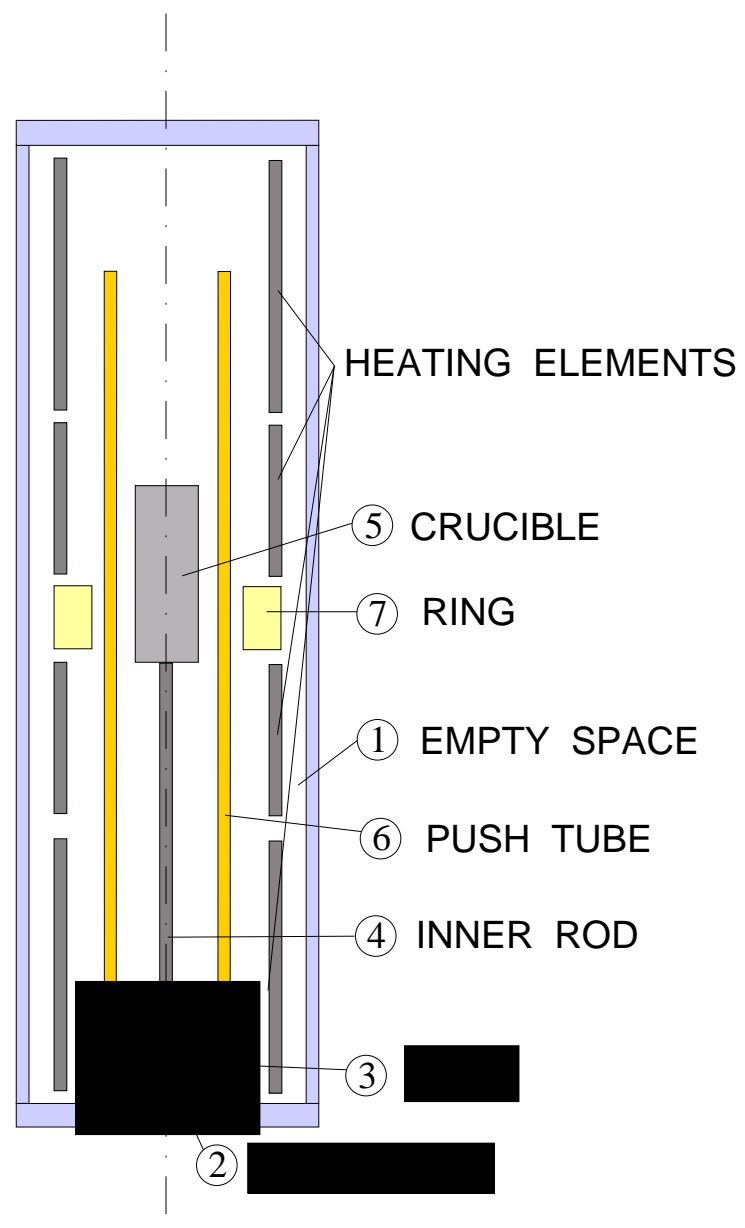

Fig. 3.1: Components of Crystal IS, Inc. furnace with regions labelled from 1 to $\%$. Courtesy of Crystal IS, Inc. 


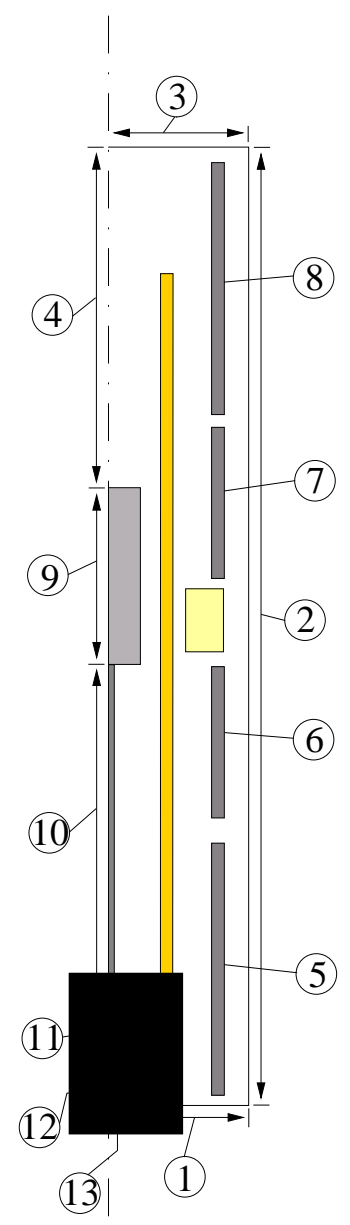

Fig. 3.2: Numbered boundary segments (surfaces) of the furnace.

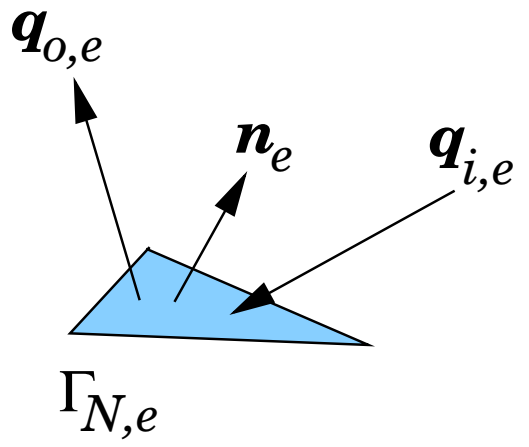

Fig. 3.3: Oriented interface patch e with outgoing and incoming radiant fluxes. 


\subsection{Gebhart's net-radiation approach}

The transport of radiant energy in the furnace is complicated by the fact that multiple reflections and re-reflexions from surfaces exposed to different temperature fields will lead to transport of energy between surface patches that are not accessible by a direct radiation beam. It would be an arduous task to follow the beams of radiation as they undergo all possible surface reflections and absorption in the furnace. The netradiation method simplifies this task considerably without introducing oversimplified assumptions. The key assumption is to consider the interfacial surfaces between the regions of interest in the furnace as composed of a suitably large number of small patches under uniform temperature with uniform heat fluxes. The patches are sufficiently small so that an individual patch is not able to "view" itself. Later we will see that in practice the patch elements are conveniently identified with curved edges of finite elements. Therefore a single finite element has to be small enough so its edge does not have appreciable curvature. We indicate spatial uniformity over the patch by dropping the dependency on $\boldsymbol{x}$ for the radiant fluxes previously defined, and by adding a bar over quantities associated to a surface patch element.

The outgoing flux in patch $\Gamma_{N, e}$ can be expressed as the sum of two positive definite contributions, namely, an emitted part and a reflected part

$$
\bar{q}_{o, e}=\sigma \epsilon_{e} \bar{T}_{e}^{4}+\rho_{e} \bar{q}_{i, e} .
$$

where $\sigma$ is the Stefan-Boltzmann constant, $\epsilon_{e}$ is the emissivity of the patch, $\rho_{e}$ is its reflectivity, and $\bar{T}_{e}^{4}$ is the average absolute temperature of the patch $\left(\bar{T}_{e}>0\right)$. Note that the reflected contribution is a $\rho_{e}$-fraction of the incoming radiant flux. Therefore, by convention $\bar{q}_{o, e}>0$ since $\rho_{e} \bar{q}_{i, e} \geq 0$. By further using the assumption $\rho_{e}=1-\epsilon_{e}$ for gray surfaces, the outgoing radiant flux reduces to

$$
\bar{q}_{o, e}=\bar{q}_{i, e}+\epsilon_{e}\left(\sigma \bar{T}_{e}^{4}-\bar{q}_{i, e}\right) .
$$

The incoming radiant energy per unit of time arriving at the patch element depends on contributions from all other existing $j$-patches as follows

$$
A_{e} \bar{q}_{i, e}=\sum_{j=1}^{N e} A_{j} F_{j \rightarrow e} \bar{q}_{o, j}
$$

where $F_{j \rightarrow e}$ is the configurational factor (view factor) between patches $j$ and $e$. This is the fraction of diffuse radiant energy that leaves the $j$ patch and arrives at the $e$ patch. It depends on the magnitude of the area of the patches and the relative orientation of them. If the patches do not view each other, the configurational factor 
is null; by definition, all of our patches have $F_{e \rightarrow e}=0$. Using the reciprocity relation $A_{j} F_{j \rightarrow e}=A_{e} F_{e \rightarrow j}$ the incoming flux of radiant energy can be rewritten as

$$
\bar{q}_{i, e}=\sum_{j=1}^{N e} F_{e \rightarrow j} \bar{q}_{o, j} .
$$

Finally, using (3.1), 3.2), (3.3), and the fact that $\sum_{j=1}^{N e} F_{e \rightarrow j}=1 \forall e$, the normal component of the net flux of radiant energy in a patch is

$$
\bar{q}_{e}=\epsilon_{e}\left(\sigma \bar{T}_{e}^{4}-\sum_{j=1}^{N e} F_{e \rightarrow j} \bar{q}_{o, j}\right) .
$$

This form of the normal net flux is inconvenient for coupled radiative-conductive heat transfer analysis. It is desirable to express the absorbed energy contribution $\epsilon_{e} \sum_{j=1}^{N e} F_{e \rightarrow j} \bar{q}_{o, j}$ as a function of the temperature of the $j$ patches and their physical properties. GEBHART (1971) has showed that

$$
\epsilon_{e} \sum_{j=1}^{N e} F_{e \rightarrow j} \bar{q}_{o, j}=\sigma \epsilon_{e} \sum_{j=1}^{N e} G_{e \rightarrow j} \bar{T}_{j}^{4}
$$

where $G_{e \rightarrow j}$ is the Gebhart factor representing the fraction of energy emitted by patch $e$ that is absorbed by patch $j$. These factors can be computed from the configuration factors and the associated reflectivity by solving the algebraic linear system of equations

$$
\begin{aligned}
& \left(\begin{array}{cccc}
\left(1-F_{1 \rightarrow 1} \rho_{1}\right) & -F_{1 \rightarrow 2} \rho_{2} & \ldots & -F_{1 \rightarrow N e} \rho_{N e} \\
-F_{2 \rightarrow 1} \rho_{1} & \left(1-F_{2 \rightarrow 2} \rho_{2}\right) & \ldots & -F_{2 \rightarrow N e} \rho_{N e} \\
\ldots \ldots \ldots \ldots \ldots \ldots \ldots \ldots \ldots \ldots \ldots & \ldots \ldots \ldots \ldots \\
-F_{N e \rightarrow 1} \rho_{1} & -F_{N e \rightarrow 2} \rho_{2} & \ldots & \left(1-F_{N e \rightarrow N e} \rho_{N e}\right)
\end{array}\right)\left(\begin{array}{c}
G_{1 \rightarrow k} \\
G_{2 \rightarrow k} \\
\ldots \\
G_{N e \rightarrow k}
\end{array}\right)=\left(\begin{array}{c}
\epsilon_{k} F_{1 \rightarrow k} \\
\epsilon_{k} F_{2 \rightarrow k} \\
\ldots \\
\epsilon_{k} F_{N e \rightarrow k}
\end{array}\right) \\
& \forall k=1, \ldots, N e \text {. }
\end{aligned}
$$

Because the matrix of coefficients is not dependent on $k$, one factorization of the system can be re-used for all remaining right side vectors in the above algebraic system. The final working formula for the normal net flux emitted on patch $e$ is

$$
\bar{q}_{e}=\sigma \epsilon_{e}\left(\bar{T}_{e}^{4}-\sum_{j=1}^{N e} G_{e \rightarrow j} \bar{T}_{j}^{4}\right) .
$$




\subsection{Galerkin-type weak formulation of heat conduction in a multiple regions furnace with discontinuities}

Let $\Omega^{\alpha}$ and $\Omega^{\beta}$ denote neighboring domains corresponding to two regions in the furnace (fig. 3.1). Let the boundary $\partial \Omega^{\alpha}$ be partitioned in three components $\partial \Omega^{\alpha}=$ $\Gamma_{D}^{\alpha} \bigcup \Gamma_{N}^{\alpha} \bigcup \Gamma^{\alpha \beta}$, where the first is the portion of $\partial \Omega^{\alpha}$ with known temperature, the second is the portion of the boundary with known flux, and the last the shared oriented interface with the neighboring region $\beta$. The subsets in the partitions do not overlap, that is $\Gamma_{D}^{\alpha} \bigcap \Gamma_{N}^{\alpha} \bigcap \Gamma^{\alpha \beta}=\emptyset$ (fig. 3.4); also, the boundary of the $\Omega^{\beta}$ region can be similarly partitioned. It is often the case that there exists a jump on the value of physical properties across the interface. Jumps on values of physical quantities such as heat fluxes are frequent particularly in the case of coupled radiative-conductive heat transfer; there will always be a discontinuity in the heat flux on the interface between an opaque region and a nonparticipating medium. In the present analysis there will be no jump on the temperature field across interfaces; the continuity of temperature across interfaces is an independent assumption.

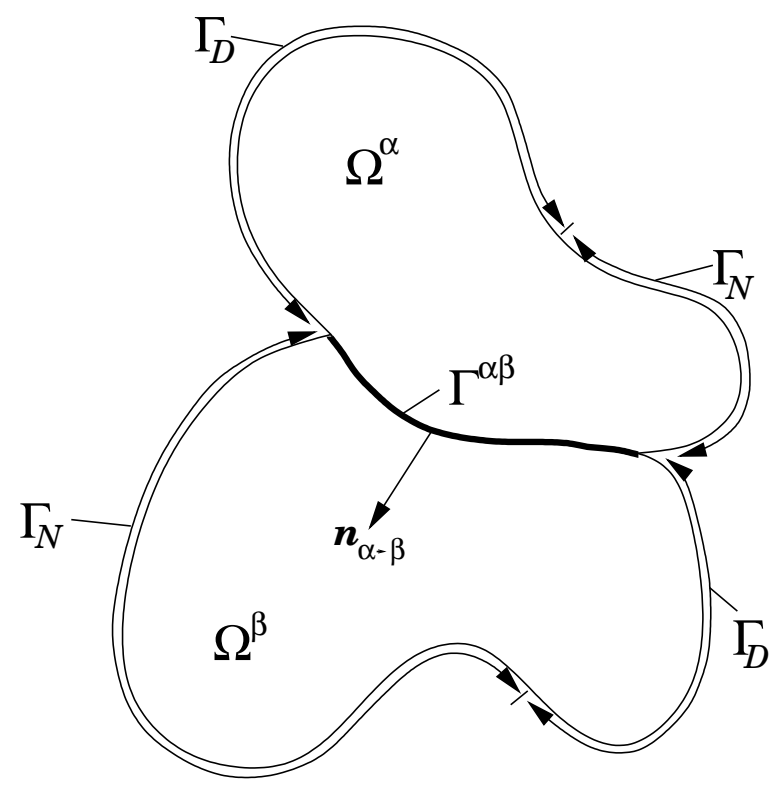

Fig. 3.4: Neighboring domains and their boundary partitions.

Let $\Omega=\bigcup_{\alpha=1}^{N r} \Omega_{\alpha}$ be the union of all $N r$ regions in the furnace (fig. 3.1), $\Gamma_{D}$, the portion of the boundary of $\Omega$ with imposed temperature, and $\Gamma_{N}$, the portion with imposed heat flux. In addition, let $\Gamma_{I}$ be the combined interface between all regions. For the geometry at hand it is observed that $\Gamma_{N}=\bigcup_{\alpha=1}^{N r} \Gamma_{N}^{\alpha}$, and $\Gamma_{D}=\bigcup_{\alpha=1}^{N r} \Gamma_{D}^{\alpha}$. It 
is desirable to find the temperature field $T: \boldsymbol{x} \rightarrow \mathbb{R} \in \Theta$ by solving the Galerkin-type weak form

$$
\mathrm{a}(T, \phi)+<\psi, \phi>_{\Gamma_{N}}+<q_{I}, \phi>_{\Gamma_{I}}=0 \quad \forall \quad \phi \in \mathrm{H}_{0}^{1}(\Omega)
$$

where

$$
\begin{aligned}
& \mathrm{a}(T, \phi):=\sum_{\alpha=1}^{N r} \int_{\Omega^{\alpha}}-k_{\alpha} \nabla T \cdot \nabla \phi d \boldsymbol{x}, \\
&<\psi, \phi>_{\Gamma_{N}}:=\sum_{\alpha=1}^{N r} \int_{\Gamma_{N}^{\alpha}} \psi^{\alpha} \phi d l, \quad \text { and } \\
&<q_{I}, \phi>_{\Gamma_{I}}:=\sum_{I=1}^{N i} \int_{\Gamma_{I}^{\alpha \beta}} q_{I}^{\alpha \rightarrow \beta} \phi d l .
\end{aligned}
$$

The trial set of solutions is $\Theta:=\left\{T \in \mathrm{H}^{1}(\Omega) \mid T=T_{D}\right.$ on $\left.\Gamma_{D}\right\}$ with $T_{D}$ the imposed temperature field on $\Gamma_{D} ; k_{\alpha}$ is the thermal conductivity of region $\alpha ; \psi^{\alpha}$ is the known normal heat flux on $\Gamma_{N}^{\alpha}$ in the direction of the outward pointing normal; $q_{I}^{\alpha \rightarrow \beta}$ is the normal heat flux across the interface $I$ between regions $\alpha$ and $\beta$ in the direction of the normal vector pointing from $\alpha$ to $\beta$; and $N i$ is the number of interfaces between regions.

The boundary segments whose union forms $\Gamma_{N}$ are those numbered $4,9,10,11$, and 12 in figure 3.2 . Those segments lie on the axis of symmetry therefore $\psi^{\alpha} \equiv 0 \forall \alpha$ and

$$
<\psi, \phi>_{\Gamma_{N}}=0 .
$$

The interface $\Gamma_{I}$ in figure 3.2 is of two kinds. Interfaces between opaque regions, and interfaces between opaque regions and the empty space. There is no discontinuity in normal flux across interfaces between opaque regions, thus

$$
q_{I}^{\alpha \rightarrow \beta}=0 \quad \text { if } \alpha \text { and } \beta \text { are opaque regions. }
$$

The resulting weak formulation becomes: find $T \in \Theta$ such that

$$
\mathrm{a}(T, \phi)+<q_{I}, \phi>_{\Gamma_{I}}=0 \quad \forall \quad \phi \in \mathrm{H}_{0}^{1}(\Omega)
$$

where

$$
<q_{I}, \phi>_{\Gamma_{I}}:=\sum_{\alpha=2}^{N r} \int_{\Gamma_{I}^{\alpha 1}} q_{I}^{\alpha \rightarrow 1} \phi d l .
$$

Oak Ridge National Laboratory Technical Report ORNL/TM-2002/64 — pp. $1-32$ 
By virtue of the definition of $\Theta$, the temperature field is continuous throughout $\Omega$ and the boundary conditions with specified temperature are automatically satisfied. There are two levels of temperature: the segments 13, 1, and 3 in figure 3.2 are at temperature $320^{\circ} \mathrm{K}$, while the segments $5,6,7$, and 8 are at $2500{ }^{\circ} \mathrm{K}$. These level of temperatures define the boundary data $T_{D}$.

The above problem is nonlinear because $q_{I}^{\alpha \rightarrow 1}$ depends nonlinearly on the temperature. Therefore an iterative scheme for solving the problem is needed. A robust scheme arises from the sequence $\{\stackrel{(n)}{T} \in \Theta \mid n=1,2, \ldots\}$, for given a suitable initial
$\stackrel{(0)}{T} \in$ $(n)$
$(n-1)$

guess $T \in \Theta$, where each iterate $T$ is obtained from its predecessor $\stackrel{(n-1)}{T} \in \Theta$ and a $(n)$

correction $T_{c} \in \mathrm{H}_{0}^{1}(\Omega)$ so that

$$
\stackrel{(n)}{T}=\stackrel{(n-1)}{T}+\stackrel{(n)}{T}
$$

where the correction satisfies

$$
\begin{aligned}
& \mathrm{a}(\stackrel{(n)}{T}, \phi)+<\left.\partial_{T} q_{I}\right|_{\substack{(n-1) \\
T}} \stackrel{(n)}{T_{c}}, \phi>_{\Gamma_{I}}=-\left(\mathrm{a}(\stackrel{(n-1)}{T}, \phi)+<\stackrel{(n-1)}{q_{I}}, \phi>_{\Gamma_{I}}\right) \\
& \forall \phi \in \mathrm{H}_{0}^{1}(\Omega) \text {. }
\end{aligned}
$$

$(n)$

Note that the latter equation is a linearization of 3.7 around $T_{c}$ wherein the mag-

nitude of the sequence of iterates, $\left\|T_{c}^{(n)}\right\|_{\mathrm{H}_{0}^{1}(\Omega)}$, converges quadratically inasmuch as the right and left sides of $(3.8 \mathrm{~b})$ are small enough for $n=1$. In the next section a systematic method for solving (3.8b) for each iterate is presented.

\subsection{Finite element approximation}

There exists a solid framework for transforming the $n$th iterate of 3.8 into a linear set of algebraic equations. The central idea is to look for a solution in a finite subspace of $\mathrm{H}_{0}^{1}(\Omega)$; this is termed a conforming method. Therefore, under certain conditions, by making the dimension of the subspace larger and larger the approximate solution may converge to the exact one. This process has been thoroughly studied (BREnNer AND ScotT, 1996; Brezzi And Fortin, 1991; Ciarlet, 1978) and here we make use of an approximation of $\mathrm{H}_{0}^{1}(\Omega)$ by means of a triangulation of $\Omega$ with quadratic polynomial basis functions. Each region of the furnace (fig. 3.4) is triangulated (figs. 3.5) so that the interface is composed of nonovelapping edges of triangles. This condition, in conjunction with a choice of polynomial space, ensures continuity of the temperature field across element boundaries and region interfaces. Next, each 
triangle is equipped with a polynomial space and a set of degrees of freedoms that represent the temperature at symmetrically spaced points within the triangle. The degrees of freedom define uniquely each element of the polynomial space and consequently the dimension of the space. The total inventory of degrees of freedom is equal to the number of algebraic equations to be solved. A finite element is a triad: a subdomain polygon, a polynomial space over the polygon, and a set of degrees of freedom.

The application of the finite element method to $(3.8)$ is standard except for the boundary radiative terms. Since the interface between all regions and the empty space is formed by edges of triangles, the radiative contribution on the right side of (3.8b) transforms into

$$
\left\langle\stackrel{(n-1)}{q_{I}}, \phi>_{\Gamma_{I}}=\sum_{\alpha=2}^{N r} \sum_{e=1}^{N e} \int_{\Gamma_{I, e}^{\alpha 1}}^{(n-1)} q_{I, e}^{\alpha \rightarrow 1} \phi d l\right.
$$

where $\Gamma_{I, e}^{\alpha 1}$ is the edge of the eth triangle on the interface between the $\alpha$ region and the empty space. Substituting GeBHART s formula (3.5) for the radiative flux on a segment yields

$$
\left\langle\stackrel{(n-1)}{q_{I}}, \phi>_{\Gamma_{I}}=\sum_{\alpha=2}^{N r} \sum_{e=1}^{N e} \int_{\Gamma_{I, e}^{\alpha 1}} \sigma \epsilon_{e}\left(\stackrel{(n-1)}{\bar{T}^{4}}-\sum_{j=1}^{N e} G_{e \rightarrow j} \stackrel{(n-1)}{\bar{T}_{j}^{4}}\right) \phi d l\right.
$$

where $\bar{T}$ denotes the average temperature along the corresponding edge of the finite element. Since $\bar{T}_{j}$ is the average temperature in all other segments visible by $\Gamma_{I, e}^{\alpha 1}$, the above formula couples explicitly the algebraic equations associated to non-neighboring finite elements. This a significant source of complexity for the assembly and solution of the resulting linear system. In addition, the fourth power term in temperature gives rise to strong non-linearity. These two features make radiative heat transfer problems difficult to solve in general.

Similarly, the sensitivity term on the left side of $(3.8 \mathrm{~b})$ can be explicitly written in terms of the average temperature along the corresponding finite element edge.

$$
<\left.\partial_{T} q_{I}\right|_{\substack{(n-1) \\ T_{c}}} \stackrel{(n)}{T_{c}}, \phi>_{\Gamma_{I}}=\sum_{\alpha=2}^{N r} \sum_{e=1}^{N e} \int_{\Gamma_{I, e}^{\alpha 1}} 4 \sigma \epsilon_{e}\left(\stackrel{(n-1)}{\bar{T}^{3}}-\sum_{j=1}^{N e} G_{e \rightarrow j} \stackrel{(n-1)}{\bar{T}_{j}^{3}}\right) \stackrel{(n)}{T_{c}} \phi d l .
$$

The following section shows results obtained with test parameters and physical properties provided by Crystal IS, Inc. 


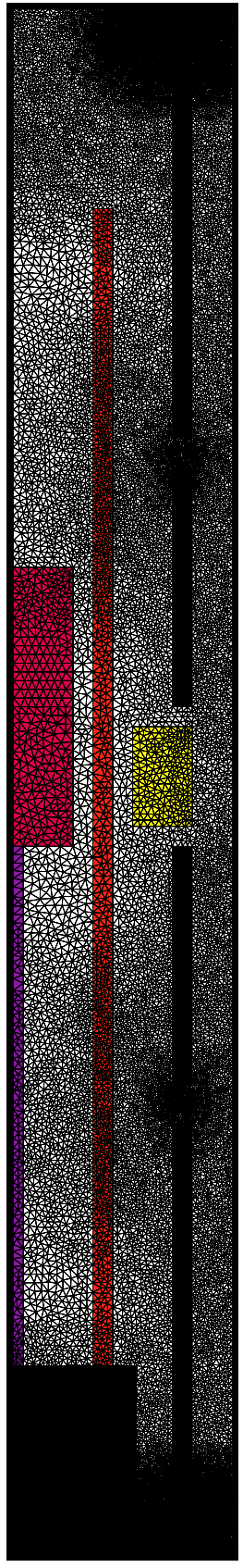

(a)

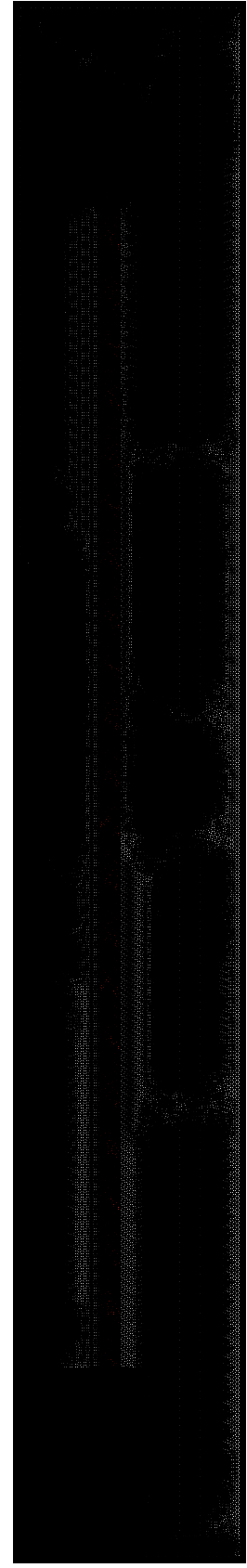

(b)

Fig. 3.5: Triangular $P_{2}$ meshes. a) 42735 triangles; total number of unknowns 15367 . b) 101442 triangles; total number of unknowns 306675. 


\subsection{Test results}

Problem (3.8) was solved (see DE Almeida AND DeRBy, 2000; DE Almeida et al., 2000, for algorithm details) with the physical properties of table 3.1. The corresponding boundary conditions at the surfaces identified in figure 3.2 are collected in table 3.2 .

Table 3.1: Fictitious physical properties for the regions of the Crystal IS, Inc. AlN single crystal growth furnace (see fig. 3.1).

\begin{tabular}{|c|c|c|}
\hline Region & $\begin{array}{c}\text { Thermal cond. } \\
(\mathrm{W} / \mathrm{m}-\mathrm{K})\end{array}$ & Emissivity $\epsilon$ \\
\hline \hline 1 & 0.1 & 0.9 \\
2 & 3 & 0.9 \\
3 & 3 & 0.9 \\
4 & 100 & 0.9 \\
5 & 100 & 0.9 \\
6 & 100 & 0.9 \\
7 & 100 & 0.9 \\
\hline
\end{tabular}

Figure 3.6 shows a typical temperature field as an elevated surface above the domain of the furnace. The figure is a top-down view showing the dimensionless temperature field, (T-320)/(2500-320), colored from blue to red on the elevated surface. The "footprints" of interfaces with discontinuity in heat flux can be clearly seen on the elevated surface. Figure 3.7 shows a similar result for a much finer triangulation. The capability of changing the physical and geometrical properties of the furnace, and quickly re-compute the temperature field is the tool sought by Crystal IS, Inc. to guide new designs of larger furnaces.

\section{Outlook}

A desirable plan for future collaboration is to augment the model (sec. 3) to include heat transfer caused by natural convection of the gases confined within the furnace. This is compelled by the fact that the scaling-up of the furnace will inevitably create larger internal void spaces allowing flow induced by density stratification to occur. The augmented model will involve an additional set of partial differential equations, 
Table 3.2: Boundary conditions of flux and temperature on the surfaces of the furnace (see fig. 3.2).

\begin{tabular}{|c|c|c|}
\hline Surface & $\begin{array}{c}\text { Temperature } \\
(\mathrm{K})\end{array}$ & $\begin{array}{c}\text { Energy flux rate } \\
\left(\mathrm{W} / \mathrm{m}^{2}\right)\end{array}$ \\
\hline \hline 1 & 320 & - \\
2 & 320 & - \\
3 & 320 & - \\
4 & - & - \\
5 & 2500 & - \\
6 & 2500 & - \\
7 & 2500 & 0 \\
8 & 2500 & 0 \\
9 & - & 0 \\
10 & - & 0 \\
11 & - & - \\
12 & - & - \\
13 & 320 & - \\
\hline
\end{tabular}




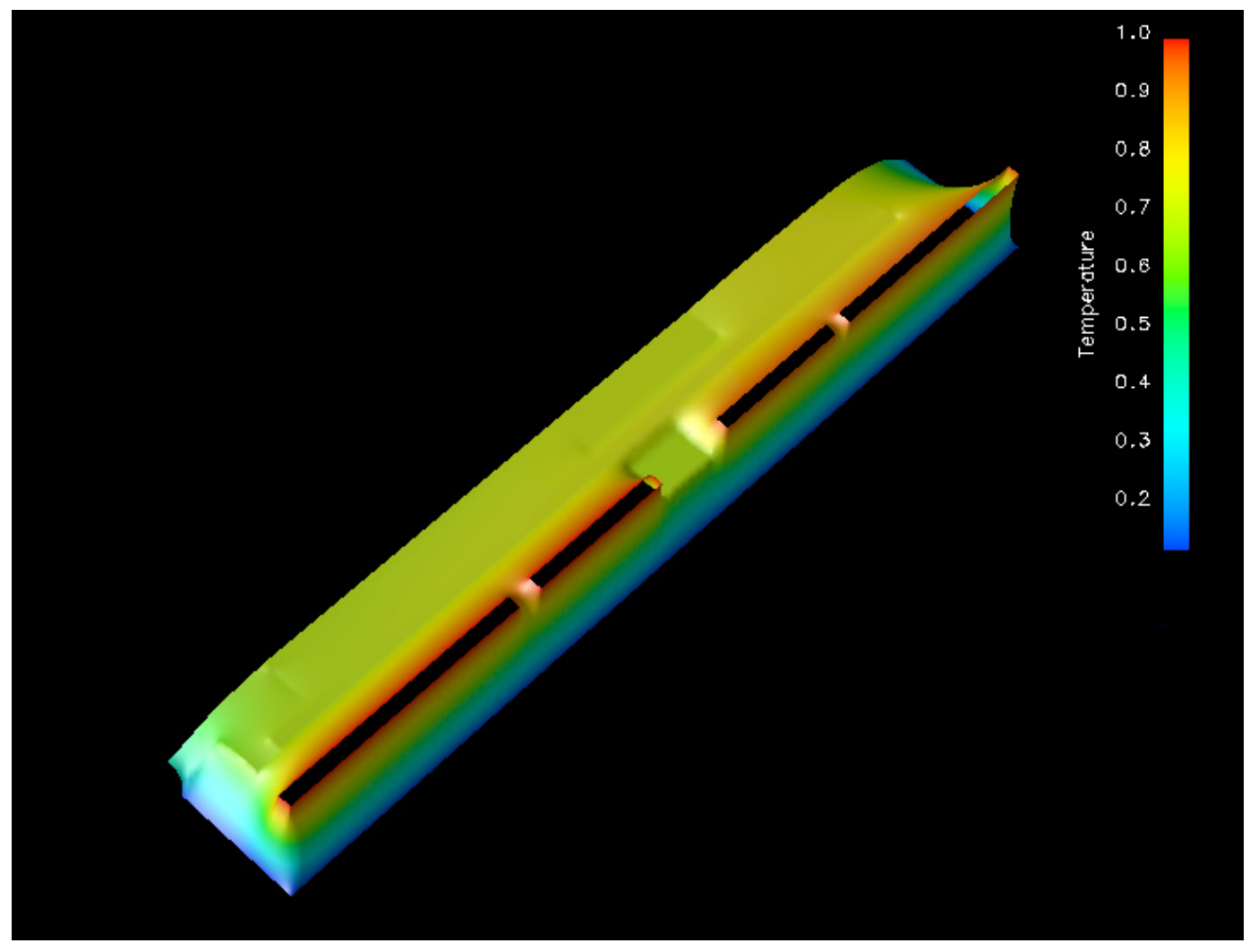

Fig. 3.6: Temperature field, (T-320)/(2500-320), with a 42 735-triangle mesh $(\epsilon=0)$. 


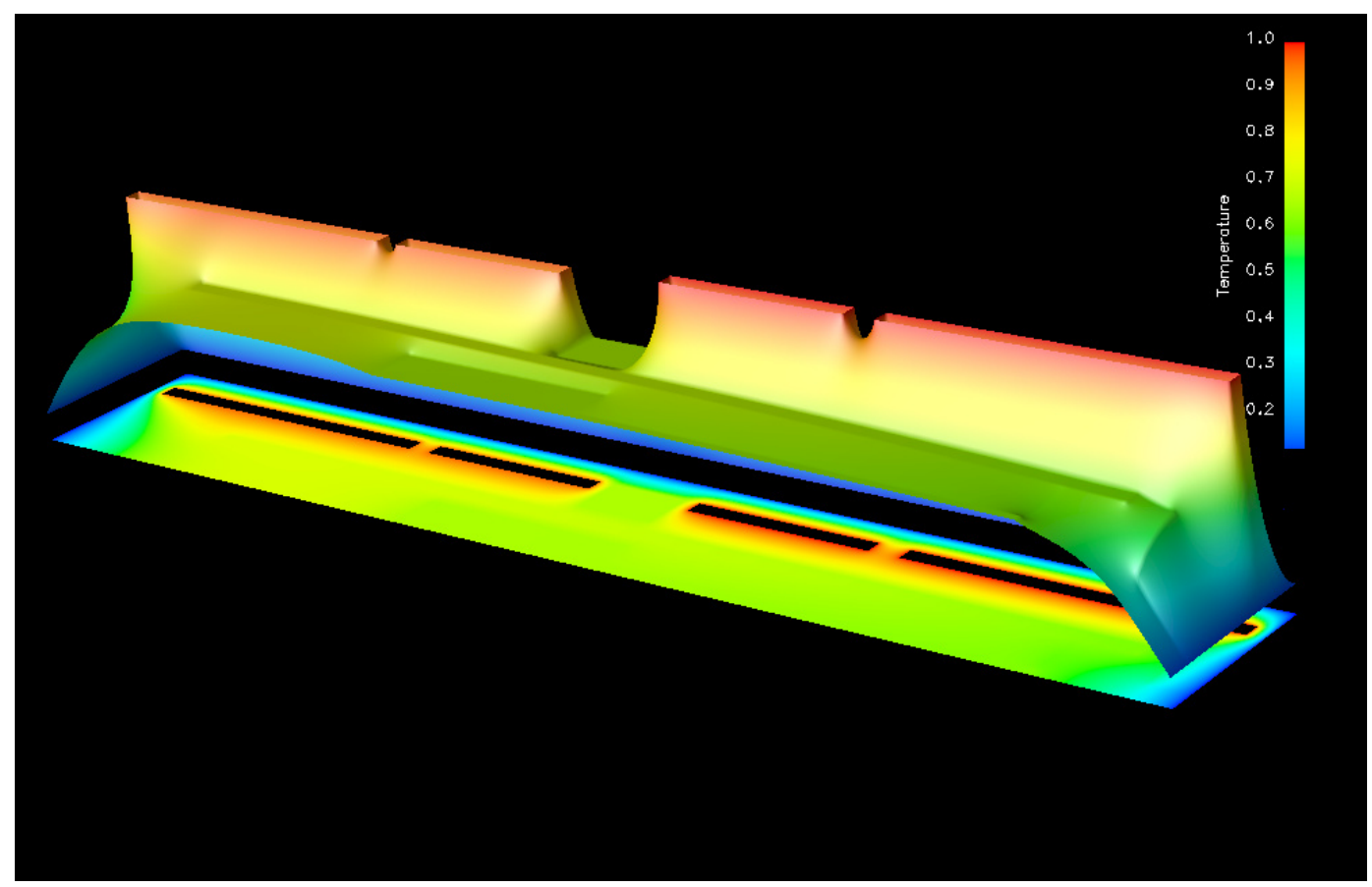

Fig. 3.7: Temperature field, (T-320)/(2500-320), with a 101442 -triangle mesh ( $\epsilon=$ $0)$. 
namely, the Navier-Stokes equations with the Boussinesq approximation in the body force term, which couples the momentum balance with heat transfer.

\title{
Acknowledgments
}

This work was supported by the Rapid Access Project (RAP) program of the Laboratory Technology Research at ORNL through the mechanism of Small CRADA ORNL-00-0587 with Crystal IS, Inc.

\author{
OAK Ridge National Laboratory \\ P.O. Box 2008 \\ OAk Ridge, Tennessee, TN 37831-6181 \\ U.S.A. \\ E-mail: dealmeidav@ornl.gov
}


Valmor de Almeida and J. Carlos Rojo

\section{References}

De Almeida, V. F. And Derby, J. J. 2000 Construction of solution curves for large 2-D problems of steady-state flows of incompressible fluids. SIAM J. Sci. Comput. 22 285-311.

de Almeida, V. F., Chapman, A. M., and Derby, J. J. 2000 On equilibration and sparse factorization of matrices arising in finite element solutions of partial differential equations. Numer. Methods Partial Different. Equ. 16 11-29.

Brenner, S. C. And Scott, L. R. 1996 The Mathematical Theory of Finite Element Methods, volume 15 of Texts in Applied Mathematics. Springer-Verlag, New York, New York.

Brezzi, F. And Fortin, M. 1991 Mixed and Hybrid Finite Element Methods, volume 15 of Computational Mathematics. Springer-Verlag, New York, New York.

Ciarlet, P. G. 1978 The Finite Element Method for Elliptic Problems. NorthHolland, Amsterdam.

Gebhart, B. 1971 Heat Tranfer. McGraw-Hill, New York.

King, S. W., Smith, L. L., Barnak, J. P., Ku, J.-H., Christman, J. A., Benjamin, M. C., Brenser, M. D., Nemanich, R. J., and Davis, R. F. 1996. Mat. Res. Soc. Symp. Proc. 395739.

Lu, H., Bhat, I., Lee, B.-C., Slack, G., And Schowalter, L. 1998 SMOCVD growth of GaN on bulk AlN substrates. Pittsburgh, MRS.

Slack, G. A. And MCNelly, T. 1976 Growth of high purity AlN crystals. J. Crystal Growth 34 263-79.

Slack, G. A. And McNelly, T. 1977 AlN single crystals. J. Crystal Growth 42 $560-3$.

Steigerwald, J., Murarka, S., and Gutmann, R. 1997 Chemical Mechanical Planarization of Microelectronic Materials. Wiley, New York. 